\title{
Sclerosing Pneumocytoma
}

National Cancer Institute

\section{Source}

National Cancer Institute. Sclerosing Pneumocytoma. NCI Thesaurus. Code C5656.

A benign tumor that arises from the lung. It is characterized by the presence of sclerotic, papillary, solid, and hemorrhagic patterns and hyperplastic type II pneumocytes.

Cholesterol clefts, hemosiderin deposition, chronic inflammation, and calcifications may be present. In the majority of cases, it is a solitary and peripheral tumor. Patients are usually asymptomatic. 